\title{
Influência do estímulo lúdico no desenvolvimento infantil diante da prática fisioterapêutica: revisão integrativa
}

\author{
Influence of ludic stimulation on child development in view of physiotherapeutic practice:
} integrative review

Influencia del estímulo del juego en el desarrollo de los niños en vista de la práctica fisioterapéutica: revisión integrativa

\section{Resumo}

O desenvolvimento infantil consiste nas transformações biológicas e comportamentais do indivíduo diante de estímulos e vivências. O momento do brincar, seja livre ou programático, permite que a criança desenvolva a realização de movimentos amplos e/ou finos, assim como o desenvolvimento da parte sensorial. O presente estudo busca investigar as evidências científicas sobre a influência de estímulo lúdico quando usado em abordagens fisioterapêuticas e a resposta diante do desenvolvimento infantil. Trata-se de uma revisão integrativa da literatura, utilizado amostra de 7 artigos selecionados a partir do levantamento de dados por meio de descritores do DeCS e MeSH: pediatria/ pediatría/ pediatrics; reabilitação/ rehabilitación/ rehabilitation; brinquedo terapêutico/ ludoterapia/ play therapy/ therapeutic play; habilidade motora/ destreza motora; brinquedo/ juego; desenvolvimento infantil; lúdico. Com essas definições, foram feitas pesquisas no SciELO, PubMED, PEDro, ScienceDirect e Google Acadêmico incluindo publicações originais nacionais e internacionais, em português, espanhol e inglês, no período de 2010 a 2020. Diante de tais pesquisas, o lúdico é um recurso simples que permite a melhor interação por parte da criança na terapia. O uso de recursos visuais auxiliam na melhora do humor e a depender da forma que é usado, pode limitar ou auxiliar nas condutas terapêuticas diante da resposta de cada criança. Diante das evidências, os estímulos lúdicos possuem um grande potencial terapêutico e são capazes de estimular pontos que até então são pouco explorados pela fisioterapia, buscando à interação e a evolução fisioterapêutica a partir de cada limitação, com recursos de fácil adaptação.

Palavras-chave: Desenvolvimento infantil; Brinquedo terapêutico; Pediatria; Fisioterapia.

\begin{abstract}
Child development consists of the individual's biological and behavioral transformations when faced with stimuli and experiences. The moment of playing, whether free or programmatic, allows the child to develop wide and/or fine movements, as well as the development of the sensory part. This study seeks to investigate the scientific evidence on the influence of playful stimulation when used in physical therapy approaches and the response to child development. This is an integrative literature review, using a sample of 7 articles selected from data collection using DeCS and MeSH descriptors: pediatrics/pediatrics/pediatrics; rehabilitation/ rehabilitation/ rehabilitation; therapeutic toy/ play therapy/ play therapy/ therapeutic play; motor skill/motor dexterity; toy/play; child development; ludic. With these definitions, searches were carried out in SciELO, PubMED, PEDro, ScienceDirect and Academic Google, including original national and international publications, in Portuguese, Spanish and English, in the period from 2010 to 2020. In the face of such research, play is a simple resource that allows the best interaction on the part of the child in
\end{abstract}


therapy. The use of visual aids helps to improve mood and, depending on the way it is used, can limit or help in therapeutic approaches to the response of each child. In view of the evidence, playful stimuli have a great therapeutic potential and are capable of stimulating points that have been little explored by physiotherapy so far, seeking interaction and physiotherapeutic evolution from each limitation, with easy-to-adapt resources.

Keywords: Child development; Therapeutic play; Pediatrics; Physiotherapy.

\section{Resumen}

El desarrollo infantil consiste en las transformaciones biológicas y conductuales del individuo ante estímulos y experiencias. El momento de juego, ya sea libre o programático, permite que el niño desarrolle movimientos amplios y / o finos, así como el desarrollo de la parte sensorial. Este estudio busca investigar la evidencia científica sobre la influencia de la estimulación lúdica cuando se usa en enfoques de fisioterapia y la respuesta al desarrollo infantil. Esta es una revisión de literatura integradora, utilizando una muestra de 7 artículos seleccionados de la recopilación de datos usando descriptores DeCS y MeSH: pediatría / pediatría / pediatría; rehabilitación / rehabilitación / rehabilitación; juguete terapéutico / terapia de juego / terapia de juego / juego terapéutico; habilidad motora / destreza motora; juguete / juego; desarrollo infantil; lúdico. Con estas definiciones, se realizaron investigaciones en SciELO, PubMED, PEDro, ScienceDirect y Academic Google, incluyendo publicaciones originales nacionales e internacionales, en portugués, español e inglés, en el período de 2010 a 2020. De cara a dicha investigación, jugar es un recurso sencillo que permite la mejor interacción por parte del niño en terapia. El uso de ayudas visuales ayuda a mejorar el estado de ánimo y, dependiendo de la forma en que se utilice, puede limitar o ayudar en los enfoques terapéuticos a la respuesta de cada niño. A la vista de la evidencia, los estímulos lúdicos tienen un gran potencial terapéutico y son capaces de estimular puntos poco explorados por la fisioterapia hasta el momento, buscando la interacción y la evolución fisioterapéutica de cada limitación, con recursos fáciles de adaptar.

Palabras clave: Desarrollo infantil; Juego terapêutico; Pediatría; Fisioterapia.

\section{Introdução}

O crescimento infantil, como um todo, possui uma evolução funcional muito rápida devido neuroplasticidade, que consiste no fácil aprendizado diante de incentivos e vivências (Lucas et al., 2016). Ao receber estímulos precoces, a criança desenvolve habilidades de forma espontânea e individualizada, e por meio das brincadeiras, é possível aumentar potencialmente o aprendizado, a criatividade e a comunicação verbal e não-verbal da criança (Silva et al., 2017b; Silva et al., 2017a; Lemos \& Veríssimo, 2020; Santos et al., 2020).

Em cada período, seja ele neonatal (0 a 28 dias de vida), lactente (29 dias a 2 anos), pré-escolar (2 a 6 anos), escolar (7 a 9 anos) ou adolescência (10 aos 19 anos) é necessário ter intervenções adequadas, seja com a utilização de brinquedos, cores, emissores sonoros, atividades de movimentação global e/ou outros artifícios contextualizados às necessidades de cada criança (Brasil, 2016; Silva et al., 2017).

Através de relatos, é mostrado que as brincadeiras transmitem valores familiares e sociais desde cedo, assim como o encorajamento para desempenhar atividades que lhes agradam e causam entusiasmo, gerando assim, indivíduos autônomos nas suas ações e tomada de decisões (Pires et al., 2015; Yogman et al. 2018). De acordo com o Estatuto da Criança e do Adolescente (ECA) referente a lei no 8.069, de 13 de julho de 1990, Cap. II - Art. 16 - "O direito à liberdade compreende os seguintes aspectos: (...) IV - brincar, praticar esportes e divertir-se.”.

O brinquedo não pode ser usado como um recurso de chantagem ou recompensa, mas como motivadores e atrativos, visto que é por meio do brincar que a criança expressa suas emoções e vivências espontaneamente, além de possuir efeito que lhe proporciona relaxamento durante atividades dolorosas e das crianças estarem diante de um momento mais descontraído e que permite que elas realizem efetivamente o tratamento (Paula et al., 2017; Silva et al., 2017a; Santos et al., 2020).

O brincar é uma terapêutica pouco compreendida e desprezada por muitos, mas quando usada de forma correta por profissionais capacitados, é possível adquirir benefícios que vão muito além da parte física, devido a humanização do tratamento (King, 2016). O progresso na infância surge diante de experiências sensoriais e/ou motoras, e o desenvolvimento motor, por sua vez, é um dos elementos mais importantes para adquirir habilidades funcionais saudáveis ao longo da vida, competências essas que são responsáveis por movimentos grossos, como caminhar, correr, saltar e ortostatismo (Lucisano et 
al., 2017).

Cabe aos profissionais adentrarem ao mundo infantil com o intuito de entender melhor as necessidades da criança a partir da sua linguagem e particularidades, uma vez que é de grande importância construir o tratamento diante do processo de ensino e executar de forma objetiva e clara para a criança (Silva et al., 2017b; Colla, 2019; Santos et al., 2020). Silva et.al. (2017) relataram que, ao utilizar jogos, brincadeiras e recursos lúdicos é notória a melhora postural, o equilíbrio, a interação, o fortalecimento de vínculos, a mobilidade e a destreza nos movimentos dos membros.

A fisioterapia pediátrica tem como finalidade avaliar, projetar e evoluir diante das necessidades da criança, e por meio do brincar terapêutico é possível observar as informações que são demonstradas (in)diretamente pelo paciente, visto que é por meio dela o profissional orienta a criança diante da atividade que está inserida ao tratamento, enquanto o brincar livre, é realizado de forma espontânea, sem regras, onde a criança segue seu enredo na atividade (Silva et al., 2017).

Em alguns casos, podem ser fatores atípicos, como o excesso ou ausência agitação, demonstram alterações ligadas ao sistema neurológico seja por consequências do desenvolvimento ou diante de influências genéticas, e ao usar tais referências para avaliação, acompanhamento e tomada de decisões perante as suas necessidades de tratamento, sejam elas de competências sociais, locomotoras e/ou intelectuais (Pereira et al., 2016; Eickmann et al., 2016; Herrero et al., 2017).

Sendo assim, o objetivo deste estudo é analisar as evidências científicas que o estímulo lúdico possui diante do desenvolvimento infantil e sua evolução de modo integral, abordando fatores motores, cognitivos, psicológicos e sociais.

\section{Metodologia}

Trata-se de uma revisão integrativa da literatura, com o objetivo de reunir resultados sobre a influência dos estímulos lúdicos no desenvolvimento infantil, a fim de abordar uma visão mais atualizada sobre o tema. A pesquisa deteve de delimitações prévias de busca, com o propósito de padronizar a coleta de artigos de forma individualizada.

Diante de pontos estabelecidos, foram realizadas buscas por descritores através do DeCS (Descritores em Ciência da Saúde) e MeSH (Medical Subject Headings) em diferentes arranjos, em português, espanhol e inglês: pediatria/ pediatria/ pediatrics; reabilitação/ rehabilitación/ rehabilitation; brinquedo terapêutico/ ludoterapia/ play therapy/ therapeutic play; habilidade motora/ destreza motora; brinquedo/ juego; desenvolvimento infantil; lúdico. Tais descritores foram usados em pesquisas na base de dados da Biblioteca Eletrônica Científica Online (SciELO), Physiotherapy Evidence Database (PEDro), PubMED, ScienceDirect e Google Acadêmico.

A coleta de dados foi de agosto de 2020 até abril de 2021, incluído ao estudo publicações originais nacionais e internacionais, em português, espanhol e inglês, do período de 2010 a 2020. Tais artigos precisavam abordar sobre o desenvolvimento infantil, a parte motora e/ou atividades e estímulos lúdicos, assim como artigos que abordam sobre a fisioterapia pediátrica.

Assim, foram excluídos artigos com mais de 10 anos de publicação a partir de 2020, revisões de literatura, relatos de caso, artigos que não fazem referência sobre a progressão do desenvolvimento infantil e pesquisas que fazem abordagem diante de outras profissões e não incluem a fisioterapia.

Diante da busca, os estudos foram excluídos quando o título já demonstrava não está de acordo com os critérios de inclusão, aqueles que eram aptos ao tema foram selecionados de forma prévia e categorizados por ano de publicação, autor e título do artigo, organizados em planilhas no Excel® e disponibilizado no Google drive, onde todas as pesquisadoras poderiam ter acesso. Em seguida, o desenvolvimento da pesquisa foi feito de forma consensual, no mesmo dia e horário por meio de reuniões no Microsoft Teams ${ }^{\circledR}$, considerando assim a leitura de todos os artigos observando os objetivos e pontos pertinentes à pesquisa. 


\section{Resultados}

Durante a coleta de dados foram encontrados diversos artigos que diante do título já foram excluídos pois não se enquadravam nos critérios de inclusão da pesquisa, e assim, obtivemos um número prévio de artigos nas bases de dados: Google Acadêmico com 58 artigos, SciELO 37 artigos, PubMed 36 artigos, PEDro e ScienceDirect nenhum artigo foi encontrado. Diante dessa seleção, foi feita a leitura dos artigos para uma análise mais criteriosa, e obteve-se uma amostra final de 7 artigos que estão de acordo com a temática dessa pesquisa. A Figura 1 mostra o processo de busca e seleção dos artigos.

Figura 1. Fluxograma de representação gráfica da aplicação dos critérios de inclusão e exclusão.

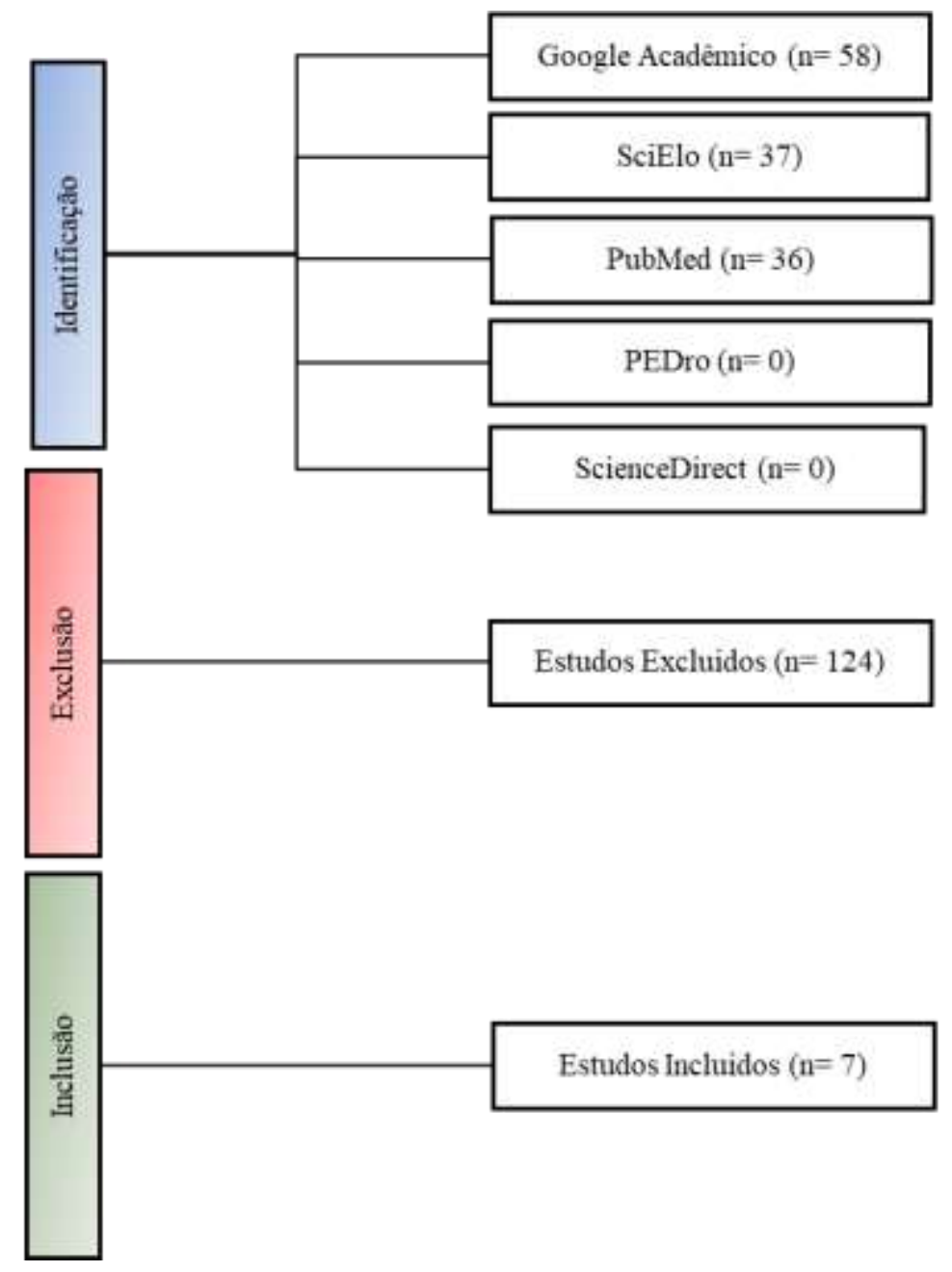

Fonte: Elaboração própria (2021).

Após a busca, seleção e análise criteriosa dos artigos, foram categorizados com relação às características gerais, como apresentado na Tabela 1 .

Tabela 1 - Descrição dos estudos a respeito do ano de publicação, tipo de estudo e amostra.

\begin{tabular}{lllc}
\hline Ano & \multicolumn{1}{c}{ Autores } & \multicolumn{1}{c}{ Tipo de estudo } & Amostra \\
\hline $\mathbf{2 0 1 8}$ & Alvarez et al. & Ensaio Clínico Randomizado & 16 \\
\hline $\mathbf{2 0 1 7}$ & Hartwig et al. & Coorte & - \\
\hline $\mathbf{2 0 1 6}$ & Sebastián et al. & Coorte & 8 \\
\hline $\mathbf{2 0 1 5}$ & Braga \& Graciani & Observacional Transversal & 10 \\
\hline $\mathbf{2 0 1 4}$ & Costa et al. & Ensaio Clínico Randomizado & 11 \\
\hline $\mathbf{2 0 1 3}$ & Hammond et al. & Ensaio Clínico Randomizado Controlado & 18 \\
\hline $\mathbf{2 0 1 1}$ & Sandlund et al. & Observacional Transversal & 14 \\
\hline
\end{tabular}

Fonte: Elaboração própria (2021). 
Research, Society and Development, v. 11, n. 1, e57311125291, 2022

(CC BY 4.0) | ISSN 2525-3409 | DOI: http://dx.doi.org/10.33448/rsd-v11i1.25291

Quanto aos tipos de estudos, três $(42,9 \%)$ foram ensaios clínicos randomizados, dois $(28,57 \%)$ foram observacionais transversais, dois (28,57\%) foram Coorte. A respeito da língua de publicação de cada artigo, três (42,9\%) foram publicados em português, três $(42,9 \%)$ em inglês e um (14,29\%) em espanhol. Na tabela 2 são apresentados os dados dos artigos a respeito dos autores, ano, títulos, objetivos, métodos e conclusões. 
Quanto aos tipos de estudos, três $(42,9 \%)$ foram ensaios clínicos randomizados, dois $(28,57 \%)$ foram observacionais transversais, dois $(28,57 \%)$ foram Coorte. A respeito da língua de publicação de cada artigo, três $(42,9 \%)$ foram publicados em português, três $(42,9 \%)$ em inglês e um $(14,29 \%)$ em espanhol. Na tabela 2 são apresentados os dados dos artigos a respeito dos autores, ano, títulos, objetivos, métodos e conclusões.

Tabela 2 - Descrição dos estudos incluídos a respeito dos autores, títulos, objetivos, métodos e conclusões.

\begin{tabular}{|c|c|c|c|c|}
\hline Autores & Título & Objetivo & Método & Conclusão \\
\hline Alvarez et al., 2018 & $\begin{array}{l}\text { Efeito de uma intervenção } \\
\text { baseada em realidade virtual nas } \\
\text { habilidades motoras básicas e no } \\
\text { controle postural de crianças com } \\
\text { Síndrome de Down }\end{array}$ & $\begin{array}{l}\text { Determinar o efeito de uma intervenção } \\
\text { baseada em realidade virtual no } \\
\text { desenvolvimento motor e no controle } \\
\text { postural de crianças com SD. }\end{array}$ & $\begin{array}{l}\text { Estudo com abordagem quantitativa, que incluiu } 16 \text { crianças com SD alocadas } \\
\text { aleatoriamente em um grupo experimental e grupo controle. O controle } \\
\text { postural foi avaliado com o TGMD- } 2 \text {. O programa de intervenção foi } \\
\text { realizado durante } 5 \text { semanas, } 2 \text { vezes/sem. Os dados foram submetidos a } \\
\text { análise estatística, teste de Shapiro Wilk para distribuição da amostra e teste t } \\
\text { de Student para comparação das médias dos grupos. }\end{array}$ & $\begin{array}{l}\text { Uma intervenção baseada em realidade virtual foi } \\
\text { eficaz apenas no grupo experimental, } \\
\text { proporcionando exercícios de baixo impacto para } \\
\text { melhorar o controle postural e, assim, causar uma } \\
\text { melhora nas habilidades motoras em crianças com } \\
\text { SD. }\end{array}$ \\
\hline $\begin{array}{l}\text { Braga \& Graciani, } \\
2015\end{array}$ & $\begin{array}{l}\text { O brincar na rotina da criança } \\
\text { com paralisia cerebral }\end{array}$ & $\begin{array}{l}\text { Verificar o perfil do brincar na rotina da } \\
\text { criança com paralisia cerebral. }\end{array}$ & $\begin{array}{l}\text { Foi realizada uma entrevista com } 10 \text { pais ou responsáveis de crianças com } \\
\text { diagnóstico de PC de ambos os sexos e com idade entre } 4 \text { e } 10 \text { anos. } \\
\text { Realizou-se uma entrevista semiestruturada por meio de } 10 \text { questões, as quais } \\
\text { abordavam o papel do brincar na rotina da criança com PC. }\end{array}$ & $\begin{array}{l}\text { Ao final do estudo, conseguiu-se observar que o } \\
\text { comprometimento motor e a consequente } \\
\text { mobilidade reduzida dificultam o manuseio de } \\
\text { objetos e o interesse. Além disso, as crianças } \\
\text { necessitam de maior incentivo e auxílio para } \\
\text { interagir e brincar. }\end{array}$ \\
\hline Costa et al., 2014 & $\begin{array}{l}\text { Recursos lúdicos aplicados na } \\
\text { clínica pediátrica do Hospital } \\
\text { Municipal de Foz do Iguaçu-PR }\end{array}$ & $\begin{array}{l}\text { Demonstrar os benefícios dos recursos } \\
\text { lúdicos na fisioterapia respiratória pediátrica. }\end{array}$ & $\begin{array}{l}\text { Trata-se de um ensaio clínico randomizado. Foram selecionadas } 11 \text { crianças } \\
\text { com diagnóstico de pneumonia e bronquite aguda, com idade variando de } 3 \\
\text { meses a } 5 \text { anos, acompanhadas de seus responsáveis legais. Os pacientes } \\
\text { foram randomizados e divididos em dois grupos homogêneos: Grupo A } \\
\text { (intervenção) e Grupo B (controle), submetidos à pré- e pós-avaliação dos } \\
\text { atendimentos. A avaliação consistiu na mensuração dos sinais vitais e na } \\
\text { aplicação de questionário. }\end{array}$ & $\begin{array}{l}\text { Os recursos lúdicos associados à fisioterapia } \\
\text { respiratória foram importantes na interação } \\
\text { fisioterapeuta-criança, mantiveram os pacientes } \\
\text { motivados e diminuíram as complicações } \\
\text { respiratórias destes. }\end{array}$ \\
\hline $\begin{array}{l}\text { Hammond et al., } \\
\qquad 2013\end{array}$ & $\begin{array}{l}\text { An investigation of the impact of } \\
\text { regular use of the Wii Fit to } \\
\text { improve motor and psychosocial } \\
\text { outcomes in children with } \\
\text { movement difficulties: a pilot } \\
\text { study }\end{array}$ & $\begin{array}{l}\text { Avaliar as sessões curtas e regulares de } \\
\text { experiência de movimento na escola usando } \\
\text { um console de videogame doméstico } \\
\text { disponível comercialmente (Nintendo Wii } \\
\text { Fit) levaria a benefícios nos domínios } \\
\text { motores e psicossociais em crianças com } \\
\text { Transtorno Dismórfico Corporal. }\end{array}$ & $\begin{array}{l}\text { Um ensaio cruzado randomizado controlado de crianças com dificuldades de } \\
\text { movimento. As crianças foram aleatoriamente designadas para uma } \\
\text { intervenção }(\mathrm{n}=10) \text { ou grupo de comparação }(\mathrm{n}=8) \text {. O grupo de intervenção } \\
\text { teve duração de } 10 \text { minutos realizando } 3 \text { vezes/sem. durante } 1 \text { mês usando o } \\
\text { Wii Fit durante o intervalo do almoço, enquanto o grupo de comparação } \\
\text { participou de seu programa normal de Jump Ahead. As avaliações pré-e pós- } \\
\text { intervenção consideraram proficiência motora, habilidade e satisfação } \\
\text { autopercebida e avaliação dos pais de problemas emocionais e } \\
\text { comportamentais. }\end{array}$ & $\begin{array}{l}\text { Este estudo fornece evidências preliminares para } \\
\text { apoiar o uso do Wii Fit em programas terapêuticos } \\
\text { para crianças com dificuldades de movimento. } \\
\text { Esta intervenção simples e popular representa um } \\
\text { método plausível para apoiar o desenvolvimento } \\
\text { motor e psicossocial das crianças. Não é possível, } \\
\text { a partir dos dados obtidos, dizer quais crianças } \\
\text { têm maior probabilidade de se beneficiar de tal } \\
\text { programa e, particularmente, qual deve ser a dose } \\
\text { e a duração. Mais pesquisas são necessárias para } \\
\text { informar essas e outras questões sobre a } \\
\text { implementação de tecnologias de realidade virtual } \\
\text { em serviços terapêuticos para crianças com } \\
\text { dificuldades de movimento. }\end{array}$ \\
\hline
\end{tabular}


Recursos lúdicos como Analisar distintas possibilidades terapêuticas Por meio de uma discussão, foi proposto o uso de brinquedos nas condutas ferramentas para fisioterapeuta

Analisar distintas possibilidades terapêuticas crianças com desenvolvimento

neuropsicomotor atípico, a partir de jogos e brinquedos terapêuticos/ pedagógicos.

Using motion interactive games Explorar a viabilidade do uso de jogos 14 crianças com PC, de 6 a 16 anos, praticaram com o EyeToy para to promote physical activity and interativos de movimento de baixo custo enhance motor performance in como uma intervenção domiciliar par children with cerebral palsy crianças com paralisia cerebral (PC)

PlayStation $2 ® \mathrm{em}$ suas casas durante 4 semanas. As medidas de resultado foram monitores de atividade física, Bateria de Avaliação de Movimento para Crianças-2, Teste de Proficiência Motora de Bruininks-Oseretsky (subteste 5: 6), Teste de Caminhada de 1 Minuto e diários de jogos.

Videogames na fisioterapia da O objetivo principal deste estudo é melhorar paralisia cerebral o desenvolvimento psicomotor da criança
com PC com o auxílio de videogames no tratamento fisioterapêutico. Participaram 8 pacientes com ICP com idade entre 6 e 12 anos, nível I-II do
E um projeto experimental, prospectivo e longitudinal com apenas um grupo. GMFCS. A intervenção fisioterapêutica utiliza o console de videogame Nintendo Wii e o jogo Wii-fit com balance board por 15 sessões. Foi estudado o equilíbrio, a distribuição da carga e a localização do centro de gravidade, bem como a motribuição da carga e a localização do centro de gravidade, durante
O trabalho apresentado reafirma a possibilidade do uso do brinquedo e dos elementos do brincar como ferramentas eficientes no trabalho desenvolvido com crianças na clínica fisioterapêutica.

É altamente viável o uso de jogos interativos de movimento na reabilitação domiciliar de crianças com PC. Os efeitos motores específicos precisam ser mais explorados.

A realidade virtual auxilia no alcance de diferentes objetivos fisioterapêuticos, sendo seu so intessante par me contro postrat Os resultados obtilos mostam que a utilização de jogos deve ser como complemento, e nunca substituto, do tratamento fisioterapêutico de crianças com PC.

Fonte: Elaboração própria (2021) 
Diante dos estudos selecionados, pode-se observar que a margem da faixa etária foi de 3 meses a 16 anos, estudando patologias como Síndrome de Down, Paralisia Cerebral, disfunções neuropsicomotoras, musculoesqueléticas, pneumonia e bronquite aguda. Nota-se que os principais auxiliadores nas terapêuticas foram o uso de estímulos sensoriais, brinquedos de fácil aquisição e uso da realidade virtual, seja no ambiente clínico, domiciliar ou hospitalar.

Os estudos feitos com base na observação transversal usam questionários, sejam com as crianças e/ou seus pais e responsáveis, já os ensaios clínicos randomizados são estudos baseados nos grupos controle e experimental, diante de sessões que foram semanais ou mensais, o Coorte, por sua vez, são discussões de casos clínicos a fim de desenvolver estudos aprofundando a temáticas e o aprimoramento de conduta.

\section{Discussão}

O referido estudo buscou avaliar, com base na literatura, a respeito da influência do estímulo lúdico no desenvolvimento infantil diante de condutas fisioterapêuticas. A partir do estudo de Hartwig et al. (2017) relatam que, atividades lúdicas possuem grande potencial terapêutico de reabilitação, além disso, o brincar se torna prazeroso na reabilitação, uma vez que estimula a relação sensorial motora das crianças com patologias neuropsicomotoras, consequentemente melhorando também a sua capacidade de se expressar.

Costa et al. (2014) evidencia que o lúdico e o brincar ajudam recém-nascidos, assim como as crianças que estão submetidas às intervenções fisioterapêuticas na área hospitalar, de tal forma que elas sejam mais colaborativas ao processo de reabilitação, ressaltando que a cada faixa etária será um método terapêutico diferente e adequado de acordo com as suas necessidades. Segundo o referido estudo, é no ambiente hospitalar que muitas vezes são estimuladas a reabilitação respiratória, sendo assim, o uso de recursos lúdicos mostrou-se com grande potencial para o desenvolvimento cardiorrespiratório.

Vale destacar também, que a síndrome do jaleco branco muitas vezes impossibilita a melhora da relação fisioterapeuta-paciente, fazendo-se necessário a busca de incentivos e aproximação com alteração nas vestimentas para cores mais chamativas e coloridas, a fim de melhorar a aceitação infantil. Será por meio da brincadeira que a criança encontrará motivação para continuar o tratamento, além de ser um recurso positivo que possibilita melhorar a relação familiar assim como terapeuta-paciente (Costa et al., 2014).

Através de Alvarez et al. (2018) é possível observar que uma das problemáticas da prática é a baixa adesão e comprometimento para realizar intervenções de reabilitação, sendo assim, o videogame surgiu com a finalidade de atrair as crianças a fim de que sejam participativas, mas, na realidade, foi muito além, se tornou possível obter participação e proporcionar um melhor bem-estar das crianças com Síndrome de Down e com outras patologias.

Sendo assim, para um melhor desenvolvimento infantil, é estimulado o equilíbrio corporal desde cedo para que apresentem um melhor desenvolvimento motor e postural, pois são submetidas a estímulos sensoriais e proprioceptivos. O uso da realidade virtual proporciona uma melhor qualidade de vida e, consequentemente, inclusão diante de diferentes faixas etárias, favorecendo assim, o desenvolvimento motor em crianças com Síndrome de Down, estimulando principalmente a mobilidade funcional (Alvarez et al.,2018)

Destaca-se no estudo de Sebastián et al. (2016) que o uso do videogame é utilizado como terapêutica para paciente com paralisia cerebral, com o intuito de melhorar o equilíbrio e o controle postural. Tais intervenções são usadas nas terapêuticas com o propósito de estimular a correção de patologias musculoesqueléticas, como escolioses e desordens de marcha, além de serem práticas dinâmicas e de fácil adaptação, favorecendo assim, o equilíbrio e a resistência contra gravitacional da criança.

Se faz necessário ter um ambiente seguro e controlado com a disponibilização de mecanismos audiovisuais, proprioceptivos com o objetivo de melhorar o feedback criança-fisioterapeuta. Vale ressaltar que a fisioterapia, quando 
estimulada desde cedo, é comprovada a existência de uma redução nos transtornos de incapacidade precoce e o uso da realidade virtual não pode ser usado como substituto do tratamento fisioterapêutico, ele pode ser utilizado como um adjuvante e ferramenta que auxilia na conduta (Sebastián et al.,2016).

No estudo realizado por Braga e Graciani (2015) com crianças com Paralisia Cerebral (PC), a maioria delas demonstram bom humor e interesse em manusear objetos e realizar atividades sozinhos, enquanto outra parte das crianças, que foram estudadas, não possuem interesses em atividades globais, como jogar bola. O brincar é atrativo para crianças com PC quando apresentam atividades com diferentes texturas, diversos aspectos visuais, como cores e figuras e alteração no peso dos brinquedos, fazendo com que a criança interaja e desenvolva melhor diante do tratamento terapêutico.

Com base nos dados obtidos no estudo de Hammond et al. (2013), as intervenções rotuladas como simples possuem suas dificuldades e capacidades de melhorar estímulos para crianças com deficiência no desenvolvimento motor e ainda assim algumas crianças relatam possuir melhora emocional e eficiência motora, mas não são todas. Tal artigo evidencia que não existe definição quanto ao tempo de duração da terapêutica lúdica, as intervenções lúdicas são escolhidas de acordo com as necessidades e respostas das crianças.

Já o artigo Sandlund et al. (2011) estudou sobre o uso da realidade virtual e evidenciou que, ao inserir a criança em um ambiente restrito, seja diante de recursos ou mobilidades, a reabilitação se torna limitada mesmo utilizando videogame como recurso auxiliar à terapêutica. Faz-se necessário ter um ambiente acolhedor para a criança e que ela sinta-se familiarizada e apta para melhor se desenvolver.

Com base nos artigos escritos por Hartwig et al. (2017), Costa et al. (2014), Sebastián et al. (2016), Hammond et al. (2013) e reforçado por King (2016), os estímulos lúdicos, sejam eles simples ou mais elaborados, possuem um grande potencial de reabilitação independente do ambiente, sejam eles domiciliar, clínico ou hospitalar. Enquanto Alvarez et al. (2018), Sebastián et al. (2016), Hammond et al. (2013) e Sandlund et al. (2011) realizaram estudos com base em jogos interativos de realidade virtual, que demonstrou ser um ótimo recurso que auxilia na interação e colaboração das crianças diante do tratamento.

Segundo os estudos de Alvarez et al. (2018), Sandlund et al. (2011) e Braga \& Graciani (2015) é de extrema importância a correlação entre a oferta de estímulos sensoriais diante da disponibilização de diferentes texturas, pesos e cores a fim de que seja trabalhado o desenvolvimento global da criança e apresentando diferentes realidades que estão fora do ambiente controlado, permitindo que ela tenha autonomia e capacidade de adaptação para melhor inclusão social, tais argumentos foram reforçadas por Silva et al. (2017) e Lucisano et al. (2017).

As condutas terapêuticas que incluem as brincadeiras possuem um destaque notável de aceitação por possuírem aspectos lúdicos, pois as crianças se sentem atraídas e desperta uma participação leve e espontânea delas (Hartwig et al., 2017; Silva et al., 2017a; Santos et al., 2020). Sobre a participação familiar, essas condutas desempenham um papel de incentivo e continuidade ao tratamento fora do ambiente clínico/ambulatorial, muitas vezes pensando na redução de deslocamento da criança, diante disso, a inclusão de brincadeiras nas condutas geram uma facilidade de adaptação ao meio, além de ser menos invasivo e de baixo custo, sendo recursos alternativos ao tratamento (Sandlund et al. 2011; Hammond et al. 2013).

Perante os estudos desenvolvidos por Hartwig et al .(2017), Sebastián et al. (2016), Alvarez et al. (2018) e Braga \& Graciani (2015) mostraram-se favorável para o uso de atividades lúdica, como brinquedos e brincadeiras inclusas nas condutas com a finalidade de reabilitação musculoesqueléticas e patologias ligadas a neuropsicomotricidade infantil, visto que desempenham estimulação para fatores globais como equilíbrio corporal, reconhecimento proprioceptivos, trabalho postural, ou mesmo atribuições mais especificas como movimentação dos dedos em pinça e lançamento de objetos, pontos esses que foram confirmados no estudo de Lucisano et al. (2017).

Nessa perspectiva, convém alencar as limitações devido à escassez de estudos atualizados nos últimos 10 anos, além 
de poucas pesquisas realizadas para a área da fisioterapia, sendo essas os maiores empecilhos para a elaboração desta revisão integrativa da literatura. Diante disso, são sugeridos novos estudos, sejam eles observacionais e/ou Coorte, a fim de desenvolver pesquisas em campo e fomentar a ciência na área da fisioterapia pediátrica, e consequentemente permitindo o desenvolvimento de novas revisões de literatura.

\section{Conclusão}

Diante das evidências obtidas, observou-se que os estímulos lúdicos possuem um grande potencial terapêutico e são capazes de estimular pontos que, até então, são poucos explorados diante da fisioterapia. Por meio dos recursos lúdicos é possível desenvolver condutas fisioterapêuticas mais atrativos que geram assim interação por parte das crianças, além de serem recursos de fácil aquisição e com adaptação de acordo com cada necessidade do paciente, e tendo como objetivo melhorar o quadro clínico a fim de adquirir o desenvolvimento infantil favorável, pois estimulam a participação e a resposta da criança diante das atividades ao qual são submetidas.

Desta forma, foi possível observar a escassez de pesquisas atualizadas nos últimos 10 anos sobre a temática diante da fisioterapia pediátrica, demonstrando assim a necessidade de mais aprofundamento do assunto. A referida revisão integrativa da literatura busca fomentar a ciência a fim de que mais estudos observacionais sejam desenvolvidos exclusivamente pela fisioterapia e/ou por meio de integração multiprofissional.

\section{Referências}

Brasil. (2015). Portaria 1.130, de 5 de Agosto de 2015. Institui a Política Nacional de Atenção Integral à Saúde da Criança (PNAISC) no âmbito do Sistema Único de Saúde (SUS). Ministério da Saúde. Secretaria de Atenção à Saúde.

Brasil. (2016). Diretrizes de estimulação precoce: crianças de zero a 3 anos com atraso no desenvolvimento neuropsicomotor decorrente de microcefalia. Ministério da Saúde. Secretaria de Atenção à Saúde.

Brasil. (2016). Lei 8.069, de 13 de Julho de 1990. Dispõe sobre o Estatuto da Criança e do Adolescente e dá outras providências. Diário Oficial da União.

Álvarez, N. G. et al. (2018). Efecto de una intervención basada en realidad virtual sobre las habi lidades motrices básicas y control postural de niños con Síndrome de Down. Revista chilena de pediatria. 89(6), 747-52.

Braga, M. A. M. \& Graciani, Z. (2015). O brincar na rotina da criança com paralisia cerebral. Cadernos de Pós-Graduação em Distúrbios do Desenvolvimento, São Paulo. 15, 41-9.

Colla, R. A. (2019). O brincar e o cuidado nos espaços da educação infantil: desenvolvendo os animais que somos. Revista Brasileira de Estudos Pedagógicos, Brasília. 100(254), 111-126.

Costa, E. F. et al. (2015). Recursos lúdicos aplicados na clínica pediátrica do Hospital Municipal de Foz do Iguaçu-PR. ConScientiae Saúde. 14 , $125-133$.

Eickmann, S. H., Emond, A. M. \& Lima, M. (2016). Avaliação do desenvolvimento infantil: além do neuromotor. Jornal de Pediatria, Porto Alegre. 92(3), 71-83.

Hammond, J. et al. (2013). An investigation of the impact of regular use of the Wii Fit to improve motor and psychosocial outcomes in children with movement difficulties: a pilot study. Child: Care, Health and Development. 40(2), 165- 175.

Hartwig, J., Geiger, D. S. \& Mayer, V. (2017). Recursos lúdicos como ferramentas para Fisioterapeutas. Salão de Ensino e Extensão, Universidade de Santa Cruz do Sul. 8 .

Herrero, D. et al. (2017). O repertório motor em lactentes de 3 a 5 meses com síndrome de Down. Research in Developmental Disabilities. 67, 1-8.

King, G. (2016). The Role of the Therapist in Therapeutic Change: How Knowledge From Mental Health Can Inform Pediatric Rehabilitation. Journal Physical \& Occupational Therapy In Pediatrics. 37(2), 121-138.

Lemos, R. A. \& Veríssimo, M. L. O. R. (2020). Estratégias metodológicas para elaboração de material educativo: em foco a promoção do desenvolvimento de prematuros. Ciência \& Saúde Coletiva, Rio de Janeiro. 25(2), 505-518.

Lucas, B. R. et al. (2016). Interventions to improve gross motor performance in children with neurodevelopmental disorders: a meta-analysis. BMC Pediatrics. 16.

Lucisano, R. V. et al. (2017). Avaliação do Brincar de "faz de 'conta” de Pré-Escolares: Revisão Integrativa da Literatura. Revista Brasileira de Educação Especial, Marília. 23(2), 309-322. 
Research, Society and Development, v. 11, n. 1, e57311125291, 2022

(CC BY 4.0) | ISSN 2525-3409 | DOI: http://dx.doi.org/10.33448/rsd-v11i1.25291

Paula, T. B. et al. (2017). Potencialidade do Lúdico como Promoção de Bem-Estar Psicológico de Pacientes em Hemodiálise. Psicologia: Ciência e Profissão, Brasília. 37, 146-158.

Pereira, K. R. G., Saccani, R. \& Valentini, N. C. (2016). Cognição e ambiente são preditores do desenvolvimento motor de bebês ao longo do tempo. Fisioterapia e Pesquisa, São Paulo. 23, 59-67.

Pires, M. R. G. M. et al. (2015). Development and validation of an instrument for evaluating the ludicity of games in health education. Revista da Escola de Enfermagem da USP, São Paulo. 49(6), 978-987.

Sandlund, M., Waterworth, E. L. \& Häger, C. (2011). Using motion interactive games to promote physical activity and enhance motor performance in children with cerebral palsy. Developmental Neurorehabilitation. 14, 15- 21.

Santos, V. L. A. et al. (2020). Understanding the dramatic therapeutic play session: a contribution to pediatric nursing. Revista Brasileira de Enfermagem, Brasília. 73(4).

Sebastián, M. P. Y. et al. (2016). Los videojuegos en el tratamiento fisioterápico de la parálisis cerebral. Fisioterapia. 38(6), $295-302$.

Silva, A. S., Valenciano, P. J. \& Fujisawa, D. S. (2017). Atividade Lúdica na Fisioterapia em Pediatria: Revisão de Literatura. Revista Brasileira de Educação Especial, Marília. 23(4), 623-636.

Silva, R. D. M. et al. (2017). Therapeutic play to prepare children for invasive procedures: a systematic review. Jornal de Pediatria, Porto Alegre. 93, 6-16.

Silva, S. G. T. et al. (2017). Influence of Therapeutic Play on the anxiety of hospitalized schoolage children: Clinical trial. Revista Brasileira de Enfermagem, Brasília. 70(6), 1244- 1249.

Yogman, M. et al. (2018). The Power of Play: A Pediatric Role in Enhancing Development in Young Children. American Academy of Pediatrics. 142 (3). 